\title{
Cognitive outcome following glioma surgery
}

\author{
Pietro Ivo D’Urso ${ }^{1}$ \\ Received: 28 June 2018 / Accepted: 5 August 2018 / Published online: 9 August 2018 \\ (C) Springer-Verlag GmbH Austria, part of Springer Nature 2018
}

Glioma surgery with intraoperative mapping is increasingly becoming a key treatment and the philosophy of glioma resection guided by functional boundaries plays a key role in obtaining good surgical results in terms of extent of resection and survival rates. This has to be balanced against the risk of post-operative cognitive deterioration.

I read with interest the paper published by Brennum and colleagues [1] on Acta Neurochirurgia in May this year. The authors have reported the results of glioma surgery with intraoperative mapping showing that $18 \%$ of the patients at 12 months has new or worsened minor or moderate deficits and only $10 \%$ has no deficits.

These results are rather significant and I do think that the authors must be commended for their honesty. The authors have shared their data regarding a heterogeneous population of patients and I do think that the results of this paper can be truly representative of what happens in most Units.

Nonetheless, there are a few points that in my opinion are not entirely clear. The authors state that adjuvant therapy is one of the factors analysed. However no breakdown of these results has been provided and I think that this would have been helpful, particularly if we consider that approximately $20 \%$ of the patients included in this study were affected by a grade III glioma.
Furthermore, in Table 3, the authors have reported the reasons for terminating the procedure and specified this for $93 \%$ of their cases; it would be interesting to know what happened to the missing $7 \%$ of patients.

Noteworthy, the authors have collected data from several health professionals and must also be commended for this effort. However, in the methods, it is not clear how they have done this. In particular, it would have been helpful to know how they have balanced scenarios where the neurological outcomes have been rated differently by different health professionals.

Notwithstanding some minor criticisms related to the methods, I do think that this paper is a real pearl in that invites gliomas surgeons to analyse the surgical outcomes from a different perspective and to avoid underestimating the risk of post-operative cognitive deficits.

\section{Reference}

1. Brennum J, Engelmann CM, Thomson JA, Skjoth-Rasmussen J (2018) Glioma surgery with intraoperative mapping - balancing the onco-functional choice. Acta Neurochir 160:1043-1050

This article is part of the Topical Collection on Tumor - Glioma

Pietro Ivo D’Urso pietrivo@me.com

1 Department of Neurosurgery, Manchester Centre for Clinical Neurosciences - Salford Royal Foundation Trust, Stott Lane, Salford M6 8HD, UK 